\title{
An Exact Solution of the Second-Order Differential Equation with the Fractional/Generalised Boundary Conditions
}

\author{
Mariusz Ciesielski (iD) ${ }^{1}$ and Tomasz Blaszczyk (iD) ${ }^{2}$ \\ ${ }^{1}$ Institute of Computer and Information Sciences, Czestochowa University of Technology, Czestochowa, Poland \\ ${ }^{2}$ Institute of Mathematics, Czestochowa University of Technology, Czestochowa, Poland \\ Correspondence should be addressed to Tomasz Blaszczyk; tomasz.blaszczyk@im.pcz.pl
}

Received 30 December 2017; Accepted 26 February 2018; Published 11 April 2018

Academic Editor: Giorgio Kaniadakis

Copyright (c) 2018 Mariusz Ciesielski and Tomasz Blaszczyk. This is an open access article distributed under the Creative Commons Attribution License, which permits unrestricted use, distribution, and reproduction in any medium, provided the original work is properly cited.

\begin{abstract}
We analysed the initial/boundary value problem for the second-order homogeneous differential equation with constant coefficients in this paper. The second-order differential equation with respect to the fractional/generalised boundary conditions is studied. We presented particular solutions to the considered problem. Finally, a few illustrative examples are shown.
\end{abstract}

\section{Introduction}

The second-order differential equations provide an important mathematical tool for modelling the phenomena occurring in dynamical systems. Examples of linear or nonlinear equations appear in almost all of the natural and engineering sciences and arise in many fields of physics.

Many scientists have studied various aspects of these problems, such as physical systems described by the Duffing equation [1], noncommutative harmonic oscillators [2], oscillators in quantum physics [3], the dynamic properties of biological oscillators [4], the analysis of single and coupled low-noise microwave oscillators [5], the Mathieu oscillator [6], the relativistic oscillator [7], or the Schrodinger type oscillator [8].

Classical differential equations are defined by using the integer order derivatives. In recent years, the class of differential equations containing fractional derivatives (known as fractional differential equations) have become an important topic. There are two approaches to obtain these types of equations. The first one is to replace the integer order derivative in classical differential equations with a fractional derivative (see, e.g., [9-14]).

The second approach is a generalisation of a method known in classical and quantum mechanics, where the differential equations are obtained from conservative Lagrangian or Hamiltonian functions. These equations are known in the literature as fractional Euler-Lagrange equations, and they contain both the left and right fractional derivatives. New mechanics models for nonconservative systems, in terms of fractional derivatives, were developed by Riewe in $[15,16]$ and extended by Klimek [17, 18] and Agrawal [19, 20]. Since then, many authors have studied the fractional differential equations of the variational type (see [21-26]).

In contrast to the above-mentioned references, where the authors analysed the integer order differential equations with classical boundary/initial conditions or fractional differential equations with the Dirichlet or natural boundary conditions, in this paper we consider the second-order differential equation with the fractional/generalised boundary conditions.

\section{Statement of the Problem}

In this paper, we solve the second-order differential equation

$$
D^{2} y(x) \pm k^{2} y(x)=0, \quad x \in[a, b], k>0
$$

with respect to the following fractional/generalised boundary conditions:

$$
\begin{gathered}
\Phi\left(\left.D_{a^{+}}^{\alpha_{1}} y(x)\right|_{x=b},\left.D_{a^{+}}^{\alpha_{2}} y(x)\right|_{x=b},\left.D_{b^{-}}^{\beta_{1}} y(x)\right|_{x=a},\right. \\
\left.\left.D_{b^{-}}^{\beta_{2}} y(x)\right|_{x=a}\right)=0,
\end{gathered}
$$


where $\alpha_{1}, \alpha_{2}, \beta_{1}, \beta_{2} \in[0,2], \alpha_{1} \neq \alpha_{2}, \beta_{1} \neq \beta_{2}$, and the operators $D_{a^{+}}^{\alpha}, D_{b^{-}}^{\alpha}$ denote the left and right RiemannLiouville derivatives, defined, respectively, by [27]

$$
\begin{aligned}
& D_{a^{+}}^{\alpha} y(x):= \begin{cases}\frac{1}{\Gamma(n-\alpha)} \frac{d^{n}}{d x^{n}} \int_{a}^{x} \frac{y(\tau)}{(x-\tau)^{\alpha-n+1}} d \tau, & \text { for } \alpha \in \mathbb{R} \backslash \mathbb{N}_{0}, n=[\alpha]+1 \\
D^{n} y(x) & \text { for } \alpha=n \in \mathbb{N}_{0}\end{cases} \\
& D_{b^{-}}^{\alpha} y(x):= \begin{cases}\frac{(-1)^{n}}{\Gamma(n-\alpha)} \frac{d^{n}}{d x^{n}} \int_{x}^{b} \frac{y(\tau)}{(\tau-x)^{\alpha-n+1}} d \tau, & \text { for } \alpha \in \mathbb{R} \backslash \mathbb{N}_{0}, n=[\alpha]+1 \\
(-1)^{n} D^{n} y(x), & \text { for } \alpha=n \in \mathbb{N}_{0}\end{cases}
\end{aligned}
$$

and $D^{n} y(x) \equiv d^{n} y(x) / d x^{n}$

Let us consider two particular cases of (1).

Case $i$. Here, we study the following differential equation:

$$
D^{2} y(x)+k^{2} y(x)=0 .
$$

It is well known that (4) has a general solution, for $k \neq 0$, given by

$$
y(x)=C_{1} \sin (k x)+C_{2} \cos (k x) .
$$

The solution (5) contains two arbitrary independent constants of integration $C_{1}$ and $C_{2}$. A particular solution can be derived from the general solution by applying the set of initial or boundary conditions.
The fractional differentiation of general solution (5) (using the left-side fractional operator) gives us

$$
\begin{aligned}
D_{a^{+}}^{\alpha} y(x) & =D_{a^{+}}^{\alpha}\left(C_{1} \sin (k x)+C_{2} \cos (k x)\right) \\
& =C_{1} D_{a^{+}}^{\alpha} \sin (k x)+C_{2} D_{a^{+}}^{\alpha} \cos (k x)
\end{aligned}
$$

and differentiation by using the right-side operator leads to

$$
\begin{aligned}
D_{b^{-}}^{\alpha} y(x) & =D_{b^{-}}^{\alpha}\left(C_{1} \sin (k x)+C_{2} \cos (k x)\right) \\
& =C_{1} D_{b^{-}}^{\alpha} \sin (k x)+C_{2} D_{b^{-}}^{\alpha} \cos (k x) .
\end{aligned}
$$

Now, we formulate the following properties for RiemannLiouville derivatives of the sine and cosine functions.

Property 1 (the left-sided Riemann-Liouville fractional derivatives of the sine and cosine functions). Let $\alpha \geq 0$ and $k \neq 0$. Then, the following relations hold:

$$
\begin{aligned}
& D_{a^{+}}^{\alpha} \sin (k x)= \begin{cases}(x-a)^{-\alpha}\left(\cos (k a) \sum_{i=0}^{\infty} \frac{(-1)^{i}(k(x-a))^{2 i+1}}{\Gamma(2 i+2-\alpha)}+\sin (k a) \sum_{i=0}^{\infty} \frac{(-1)^{i}(k(x-a))^{2 i}}{\Gamma(2 i+1-\alpha)}\right) & \text { for } \alpha>0 \wedge \alpha \notin \mathbb{N}_{0} \\
k^{n} \sin \left(k x+\frac{n \pi}{2}\right) & \text { for } \alpha=n \in \mathbb{N}_{0}\end{cases} \\
& D_{a^{+}}^{\alpha} \cos (k x)= \begin{cases}(x-a)^{-\alpha}\left(\cos (k a) \sum_{i=0}^{\infty} \frac{(-1)^{i}(k(x-a))^{2 i}}{\Gamma(2 i+1-\alpha)}-\sin (k a) \sum_{i=0}^{\infty} \frac{(-1)^{i}(k(x-a))^{2 i+1}}{\Gamma(2 i+2-\alpha)}\right) & \text { for } \alpha>0 \wedge \alpha \notin \mathbb{N}_{0} \\
k^{n} \cos \left(k x+\frac{n \pi}{2}\right) & \text { for } \alpha=n \in \mathbb{N}_{0} .\end{cases}
\end{aligned}
$$

Property 2 (the right-sided Riemann-Liouville fractional derivatives of the sine and cosine functions). Let $\alpha \geq 0$ and $k \neq 0$. Then, the following relations hold:

$$
\begin{aligned}
& D_{b^{-}}^{\alpha} \sin (k x)= \begin{cases}(b-x)^{-\alpha}\left(\cos (k b) \sum_{i=0}^{\infty} \frac{(-1)^{i}(k(b-x))^{2 i}}{\Gamma(2 i+1-\alpha)}+\sin (k b) \sum_{i=0}^{\infty} \frac{(-1)^{i}(k(b-x))^{2 i+1}}{\Gamma(2 i+2-\alpha)}\right) & \text { for } \alpha>0 \wedge \alpha \notin \mathbb{N}_{0} \\
(-1)^{n} k^{n} \sin \left(k x+\frac{n \pi}{2}\right) & \text { for } \alpha=n \in \mathbb{N}_{0}\end{cases} \\
& D_{b^{-}}^{\alpha} \cos (k x)= \begin{cases}(b-x)^{-\alpha}\left(\cos (k b) \sum_{i=0}^{\infty} \frac{(-1)^{i}(k(b-x))^{2 i}}{\Gamma(2 i+1-\alpha)}+\sin (k b) \sum_{i=0}^{\infty} \frac{(-1)^{i}(k(b-x))^{2 i+1}}{\Gamma(2 i+2-\alpha)}\right) & \text { for } \alpha>0 \wedge \alpha \notin \mathbb{N}_{0} \\
(-1)^{n} k^{n} \cos \left(k x+\frac{n \pi}{2}\right) & \text { for } \alpha=n \in \mathbb{N}_{0} .\end{cases}
\end{aligned}
$$


Proof (Properties 1 and 2). We use Taylor's series expansions of sine and cosine functions [28]

$$
\begin{aligned}
& \sin (x)=\sum_{i=0}^{\infty} \frac{(-1)^{i} x^{2 i+1}}{\Gamma(2 i+2)}, \\
& \cos (x)=\sum_{i=0}^{\infty} \frac{(-1)^{i} x^{2 i}}{\Gamma(2 i+1)}
\end{aligned}
$$

and properties of the left and right-sided fractional derivatives of power functions [27]

$$
\begin{aligned}
D_{a^{+}}^{\alpha}(x-a)^{m} & =\frac{\Gamma(m+1)}{\Gamma(m+1-\alpha)}(x-a)^{m-\alpha}, \\
\alpha \geq 0, m>-1 & \\
D_{b^{-}}^{\alpha}(b-x)^{m} & =\frac{\Gamma(m+1)}{\Gamma(m+1-\alpha)}(b-x)^{m-\alpha},
\end{aligned}
$$

$$
\alpha \geq 0, m>-1 \text {. }
$$

Also we apply the known fundamental trigonometric identities

$$
\begin{aligned}
\sin (k x)= & \sin (k(x-a)+k a) \\
= & \sin (k(x-a)) \cos (k a) \\
& +\cos (k(x-a)) \sin (k a) \\
\cos (k x)= & \cos (k(x-a)+k a) \\
= & \cos (k(x-a)) \cos (k a) \\
& -\sin (k(x-a)) \sin (k a) \\
\sin (k x)=- & \sin (k(b-x)-k b) \\
= & -\sin (k(b-x)) \cos (k b) \\
& +\cos (k(b-x)) \sin (k b) \\
\cos (k x)= & \cos (k(b-x)-k b) \\
= & \cos (k(b-x)) \cos (k b) \\
& +\sin (k(b-x)) \sin (k b) .
\end{aligned}
$$

Then

$$
\begin{aligned}
D_{a^{+}}^{\alpha} \sin (k x)= & \cos (k a) D_{a^{+}}^{\alpha} \sin (k(x-a)) \\
& +\sin (k a) D_{a^{+}}^{\alpha} \cos (k(x-a)) \\
D_{a^{+}}^{\alpha} \cos (k x)= & \cos (k a) D_{a^{+}}^{\alpha} \cos (k(x-a)) \\
& -\sin (k a) D_{a^{+}}^{\alpha} \sin (k(x-a)) \\
D_{b^{-}}^{\alpha} \sin (k x)= & -\cos (k b) D_{b^{-}}^{\alpha} \sin (k(b-x)) \\
& +\sin (k b) D_{b^{-}}^{\alpha} \cos (k(b-x)) \\
D_{b^{-}}^{\alpha} \cos (k x)= & \cos (k b) D_{b^{-}}^{\alpha} \cos (k(b-x)) \\
& +\sin (k b) D_{b^{-}}^{\alpha} \sin (k(b-x)),
\end{aligned}
$$

where

$$
\begin{aligned}
D_{a^{+}}^{\alpha} & \sin (k(x-a)) \\
& =D_{a^{+}}^{\alpha}\left(\sum_{i=0}^{\infty} \frac{(-1)^{i}}{\Gamma(2 i+2)}(k(x-a))^{2 i+1}\right) \\
& =\sum_{i=0}^{\infty} \frac{(-1)^{i} k^{2 i+1}}{\Gamma(2 i+2)} D_{a^{+}}^{\alpha}(x-a)^{2 i+1} \\
& =\sum_{i=0}^{\infty} \frac{(-1)^{i} k^{2 i+1}}{\Gamma(2 i+2-\alpha)}(x-a)^{2 i+1-\alpha} \\
& =(x-a)^{-\alpha} \sum_{i=0}^{\infty} \frac{(-1)^{i}(k(x-a))^{2 i+1}}{\Gamma(2 i+2-\alpha)}
\end{aligned}
$$

and in a similar way we obtain

$$
\begin{aligned}
& D_{a^{+}}^{\alpha} \cos (k(x-a))=(x-a)^{-\alpha} \sum_{i=0}^{\infty} \frac{(-1)^{i}(k(x-a))^{2 i}}{\Gamma(2 i+1-\alpha)} \\
& D_{b^{-}}^{\alpha} \sin (k(b-x)) \\
& \quad=(b-x)^{-\alpha} \sum_{i=0}^{\infty} \frac{(-1)^{i}(k(b-x))^{2 i+1}}{\Gamma(2 i+2-\alpha)} \\
& D_{b^{-}}^{\alpha} \cos (k(b-x))=(b-x)^{-\alpha} \sum_{i=0}^{\infty} \frac{(-1)^{i}(k(b-x))^{2 i}}{\Gamma(2 i+1-\alpha)} .
\end{aligned}
$$

Finally, putting (17)-(20) into (13)-(16), we obtain the formulas in Properties 1 and 2.

Remark 3. Note that the infinite series included in formulas (17)-(20) can be expressed by formulas containing the Mittag-Leffler function. This observation leads us to the following expressions:

$$
\begin{aligned}
& D_{a^{+}}^{\alpha} \sin (k(x-a)) \\
& \quad=k(x-a)^{1-\alpha} E_{2,2-\alpha}\left(-k^{2}(x-a)^{2}\right) \\
& D_{a^{+}}^{\alpha} \cos (k(x-a))=(x-a)^{-\alpha} E_{2,1-\alpha}\left(-k^{2}(x-a)^{2}\right) \\
& D_{b^{-}}^{\alpha} \sin (k(b-x)) \\
& \quad=k(b-x)^{1-\alpha} E_{2,2-\alpha}\left(-k^{2}(b-x)^{2}\right) \\
& D_{b^{-}}^{\alpha} \cos (k(b-x))=(b-x)^{-\alpha} E_{2,1-\alpha}\left(-k^{2}(b-x)^{2}\right),
\end{aligned}
$$

where $E_{p, q}$ denotes the Mittag-Leffler function defined in [27]

$$
E_{p, q}(x)=\sum_{i=0}^{\infty} \frac{x^{i}}{\Gamma(p i+q)}, \quad p, q \in \mathbb{R}, p>0, x \in \mathbb{R} .
$$

The above-mentioned notations can be useful in case when one uses the built-in function (the Mittag-Leffler function) in a mathematical software. 
Case ii. The second problem has the following form:

$$
D^{2} y(x)-k^{2} y(x)=0 .
$$

In this case the general solution of (23), for $k \neq 0$, is given by

$$
y(x)=C_{1} \sinh (k x)+C_{2} \cosh (k x) .
$$

The fractional differentiation of solution (24) (using the leftside operator) gives

$$
\begin{aligned}
D_{a^{+}}^{\alpha} y(x) & =D_{a^{+}}^{\alpha}\left(C_{1} \sinh (k x)+C_{2} \cosh (k x)\right) \\
& =C_{1} D_{a^{+}}^{\alpha} \sinh (k x)+C_{2} D_{a^{+}}^{\alpha} \cosh (k x)
\end{aligned}
$$

and for the right-side derivative we have

$$
\begin{aligned}
D_{b^{-}}^{\alpha} y(x) & =D_{b^{-}}^{\alpha}\left(C_{1} \sinh (k x)+C_{2} \cosh (k x)\right) \\
& =C_{1} D_{b^{-}}^{\alpha} \sinh (k x)+C_{2} D_{b^{-}}^{\alpha} \cosh (k x) .
\end{aligned}
$$

Next, we formulate the following properties for RiemannLiouville derivatives of the hyperbolic sine and hyperbolic cosine functions.

Property 4 (the left-sided Riemann-Liouville fractional derivatives of the hyperbolic sine and hyperbolic cosine functions). Let $\alpha \geq 0$ and $k \neq 0$. Then, the following relations hold:

$$
\begin{aligned}
& D_{a^{+}}^{\alpha} \sinh (k x)= \begin{cases}(x-a)^{-\alpha}\left(\cosh (k a) \sum_{i=0}^{\infty} \frac{(k(x-a))^{2 i+1}}{\Gamma(2 i+2-\alpha)}+\sinh (k a) \sum_{i=0}^{\infty} \frac{(k(x-a))^{2 i}}{\Gamma(2 i+1-\alpha)}\right) & \text { for } \alpha>0 \wedge \alpha \notin \mathbb{N}_{0} \\
k^{n} \begin{cases}\cosh (k x) & \text { for } n=1,3,5, \ldots \\
\sinh (k x) & \text { for } n=0,2,4, \ldots\end{cases} & \text { for } \alpha=n \in \mathbb{N}_{0} .\end{cases} \\
& D_{a^{+}}^{\alpha} \cosh (k x)= \begin{cases}(x-a)^{-\alpha}\left(\cosh (k a) \sum_{i=0}^{\infty} \frac{(k(x-a))^{2 i}}{\Gamma(2 i+1-\alpha)}+\sinh (k a) \sum_{i=0}^{\infty} \frac{(k(x-a))^{2 i+1}}{\Gamma(2 i+2-\alpha)}\right) & \text { for } \alpha>0 \wedge \alpha \notin \mathbb{N}_{0} \\
k^{n} \begin{cases}\sinh (k x) & \text { for } n=1,3,5, \ldots \\
\cosh (k x) & \text { for } n=0,2,4, \ldots\end{cases} & \text { for } \alpha=n \in \mathbb{N}_{0}\end{cases}
\end{aligned}
$$

Property 5 (the right-sided Riemann-Liouville fractional derivatives of the hyperbolic sine and hyperbolic cosine functions). Let $\alpha \geq 0$ and $k \neq 0$. Then, the following relations hold:

$$
\begin{aligned}
& D_{b^{-}}^{\alpha} \sinh (k x)= \begin{cases}(b-x)^{-\alpha}\left(-\cosh (k b) \sum_{i=0}^{\infty} \frac{(k(b-x))^{2 i+1}}{\Gamma(2 i+2-\alpha)}+\sinh (k b) \sum_{i=0}^{\infty} \frac{(k(b-x))^{2 i}}{\Gamma(2 i+1-\alpha)}\right) & \text { for } \alpha>0 \wedge \alpha \notin \mathbb{N}_{0} \\
(-1)^{n} k^{n} \begin{cases}\cosh (k x) & \text { for } n=1,3,5, \ldots \\
\sinh (k x) & \text { for } n=0,2,4, \ldots\end{cases} & \text { for } \alpha=n \in \mathbb{N}_{0}\end{cases} \\
& D_{b^{-}}^{\alpha} \cosh (k x)= \begin{cases}(b-x)^{-\alpha}\left(\cosh (k b) \sum_{i=0}^{\infty} \frac{(k(b-x))^{2 i}}{\Gamma(2 i+1-\alpha)}-\sinh (k b) \sum_{i=0}^{\infty} \frac{(k(b-x))^{2 i+1}}{\Gamma(2 i+2-\alpha)}\right) & \text { for } \alpha>0 \wedge \alpha \notin \mathbb{N}_{0} \\
(-1)^{n} k^{n} \begin{cases}\sinh (k x) & \text { for } n=1,3,5, \ldots \\
\cosh (k x) & \text { for } n=0,2,4, \ldots\end{cases} & \text { for } \alpha=n \in \mathbb{N}_{0} .\end{cases}
\end{aligned}
$$

Proof (Properties 4 and 5). Here we apply Taylor's series expansions of the hyperbolic sine and cosine functions [28]

$$
\begin{aligned}
& \sinh (x)=\sum_{i=0}^{\infty} \frac{x^{2 i+1}}{\Gamma(2 i+2)} \\
& \cosh (x)=\sum_{i=0}^{\infty} \frac{x^{2 i}}{\Gamma(2 i+1)}
\end{aligned}
$$

and the trigonometric identities

$$
\begin{aligned}
\sinh (k x)= & \sinh (k(x-a)+k a) \\
= & \sinh (k(x-a)) \cosh (k a) \\
& +\cosh (k(x-a)) \sinh (k a) \\
\cosh (k x)= & \cosh (k(x-a)+k a) \\
= & \cosh (k(x-a)) \cosh (k a) \\
& +\sinh (k(x-a)) \sinh (k a)
\end{aligned}
$$




$$
\begin{aligned}
\sinh (k x)= & -\sinh (k(b-x)-k b) \\
= & -\sinh (k(b-x)) \cosh (k b) \\
& +\cosh (k(b-x)) \sinh (k b) \\
\cosh (k x)= & \cosh (k(b-x)-k b) \\
= & \cosh (k(b-x)) \cosh (k b) \\
& -\sinh (k(b-x)) \sinh (k b) .
\end{aligned}
$$

Then

$$
\begin{aligned}
D_{a^{+}}^{\alpha} \sinh (k x)= & \cosh (k a) D_{a^{+}}^{\alpha} \sinh (k(x-a)) \\
& +\sinh (k a) D_{a^{+}}^{\alpha} \cosh (k(x-a)) \\
D_{a^{+}}^{\alpha} \cosh (k x)= & \cosh (k a) D_{a^{+}}^{\alpha} \cosh (k(x-a)) \\
& +\sinh (k a) D_{a^{+}}^{\alpha} \sinh (k(x-a)) \\
D_{b^{-}}^{\alpha} \sinh (k x)= & -\cosh (k b) D_{b^{-}}^{\alpha} \sinh (k(b-x)) \\
& +\sinh (k b) D_{b^{-}}^{\alpha} \cosh (k(b-x)) \\
D_{b^{-}}^{\alpha} \cosh (k x)= & \cosh (k b) D_{b^{-}}^{\alpha} \cosh (k(b-x)) \\
& -\sinh (k b) D_{b^{-}}^{\alpha} \sinh (k(b-x)),
\end{aligned}
$$

where

$$
\begin{aligned}
& D_{a^{+}}^{\alpha} \sinh (k(x-a))=(x-a)^{-\alpha} \sum_{i=0}^{\infty} \frac{(k(x-a))^{2 i+1}}{\Gamma(2 i+2-\alpha)} \\
& D_{a^{+}}^{\alpha} \cosh (k(x-a))=(x-a)^{-\alpha} \sum_{i=0}^{\infty} \frac{(k(x-a))^{2 i}}{\Gamma(2 i+1-\alpha)} \\
& D_{b^{-}}^{\alpha} \sinh (k(b-x))=(b-x)^{-\alpha} \sum_{i=0}^{\infty} \frac{(k(b-x))^{2 i+1}}{\Gamma(2 i+2-\alpha)} \\
& D_{b^{-}}^{\alpha} \cosh (k(b-x))=(b-x)^{-\alpha} \sum_{i=0}^{\infty} \frac{(k(b-x))^{2 i}}{\Gamma(2 i+1-\alpha)} .
\end{aligned}
$$

Finally, putting (32) into (31), we obtain the formulas in Properties 4 and 5.
Remark 6. In formulas (32), the infinite series can be also expressed by using the Mittag-Leffler function, and we obtain

$$
\begin{aligned}
& D_{a^{+}}^{\alpha} \sinh (k(x-a)) \\
& \quad=k(x-a)^{1-\alpha} E_{2,2-\alpha}\left(k^{2}(x-a)^{2}\right) \\
& D_{a^{+}}^{\alpha} \cosh (k(x-a))=(x-a)^{-\alpha} E_{2,1-\alpha}\left(k^{2}(x-a)^{2}\right) \\
& D_{b^{-}}^{\alpha} \sinh (k(b-x)) \\
& \quad=k(b-x)^{1-\alpha} E_{2,2-\alpha}\left(k^{2}(b-x)^{2}\right)
\end{aligned}
$$

$D_{b^{-}}^{\alpha} \cosh (k(b-x))=(b-x)^{-\alpha} E_{2,1-\alpha}\left(k^{2}(b-x)^{2}\right)$.

\section{Examples of the Determination of Particular Solutions}

The boundary conditions, written in the general form (2), can be used in many combinations. Now, we show three selected examples. Other combinations of the particular boundary conditions can be easily adopted by the reader (in a similar way).

Example 7. Equation (4) with the following boundary conditions given on both sides of the domain:

$$
\left.D_{b^{-}}^{\beta_{1}} y(x)\right|_{x=a}=\left.L_{1} \wedge D_{a^{+}}^{\alpha_{1}} y(x)\right|_{x=b}=L_{2}
$$

We substitute the general solution (5) into (34) and we have

$$
\begin{aligned}
& \left.C_{1} D_{b^{-}}^{\beta_{1}} \sin (k x)\right|_{x=a}+\left.C_{2} D_{b^{-}}^{\beta_{1}} \cos (k x)\right|_{x=a}=L_{1} \\
& \left.C_{1} D_{a^{+}}^{\alpha_{1}} \sin (k x)\right|_{x=b}+\left.C_{2} D_{a^{+}}^{\alpha_{1}} \cos (k x)\right|_{x=b}=L_{2} .
\end{aligned}
$$

The independent constants of integration $C_{1}$ and $C_{2}$ can be determined from the solution of the following system of equations:

$$
\begin{aligned}
{\left[\begin{array}{l}
C_{1} \\
C_{2}
\end{array}\right]=} & {\left[\begin{array}{ll}
\left.D_{b^{-}}^{\beta_{1}} \sin (k x)\right|_{x=a} & \left.D_{b^{-}}^{\beta_{1}} \cos (k x)\right|_{x=a} \\
\left.D_{a^{+}}^{\alpha_{1}} \sin (k x)\right|_{x=b} & \left.D_{a^{+}}^{\alpha_{1}} \cos (k x)\right|_{x=b}
\end{array}\right]^{-1} } \\
& \cdot\left[\begin{array}{c}
L_{1} \\
L_{2}
\end{array}\right] .
\end{aligned}
$$

The analytical solution of (36) is of the form

$$
\begin{aligned}
& C_{1}=\frac{\left.L_{1} D_{a^{+}}^{\alpha_{1}} \cos (k x)\right|_{x=b}-\left.L_{2} D_{b^{-}}^{\beta_{1}} \cos (k x)\right|_{x=a}}{\left.\left.D_{b^{-}}^{\beta_{1}} \sin (k x)\right|_{x=a} \cdot D_{a^{+}}^{\alpha_{1}} \cos (k x)\right|_{x=b}-\left.\left.D_{a^{+}}^{\alpha_{1}} \sin (k x)\right|_{x=b} \cdot D_{b^{-}}^{\beta_{1}} \cos (k x)\right|_{x=a}} \\
& C_{2}=\frac{-\left.L_{1} D_{a^{+}}^{\alpha_{1}} \sin (k x)\right|_{x=b}+\left.L_{2} D_{b^{-}}^{\beta_{1}} \sin (k x)\right|_{x=a}}{\left.\left.D_{b^{-}}^{\beta_{1}} \sin (k x)\right|_{x=a} \cdot D_{a^{+}}^{\alpha_{1}} \cos (k x)\right|_{x=b}-\left.\left.D_{a^{+}}^{\alpha_{1}} \sin (k x)\right|_{x=b} \cdot D_{b^{-}}^{\beta_{1}} \cos (k x)\right|_{x=a}} .
\end{aligned}
$$


Example 8. Equation (23) with the conditions given on the left side of the domain $(x=a)$ : this case corresponds to the initial value problem

$$
\left.D_{b^{-}}^{\beta_{1}} y(x)\right|_{x=a}=\left.L_{1} \wedge D_{b^{-}}^{\beta_{2}} y(x)\right|_{x=a}=L_{2}, \quad \beta_{1} \neq \beta_{2} .
$$

When we put the general solution (24) into (38), then we obtain the following linear system of equations:

$$
\begin{aligned}
& \left.C_{1} D_{b^{-}}^{\beta_{1}} \sinh (k x)\right|_{x=a}+\left.C_{2} D_{b^{-}}^{\beta_{1}} \cosh (k x)\right|_{x=a}=L_{1} \\
& \left.C_{1} D_{b^{-}}^{\beta_{2}} \sinh (k x)\right|_{x=a}+\left.C_{2} D_{b^{-}}^{\beta_{2}} \cosh (k x)\right|_{x=a}=L_{2}
\end{aligned}
$$

that can be also written in the matrix form

$$
\begin{aligned}
{\left[\begin{array}{l}
C_{1} \\
C_{2}
\end{array}\right]=} & {\left[\begin{array}{cc}
\left.D_{b^{-}}^{\beta_{1}} \sinh (k x)\right|_{x=a} & \left.D_{b^{-}}^{\beta_{1}} \cosh (k x)\right|_{x=a} \\
\left.D_{b^{-}}^{\beta_{2}} \sinh (k x)\right|_{x=b} & \left.D_{b^{-}}^{\beta_{2}} \cosh (k x)\right|_{x=a} ^{-1}
\end{array}\right]^{-1} } \\
& \cdot\left[\begin{array}{c}
L_{1} \\
L_{2}
\end{array}\right] .
\end{aligned}
$$

$$
\left[\begin{array}{c}
C_{1} \\
C_{2}
\end{array}\right]=\left[\begin{array}{c}
\left.\mu_{1} D_{b^{-}}^{\beta_{1}} \sin (k x)\right|_{x=a}+\left.\mu_{2} D_{b^{-}}^{\beta_{2}} \sin (k x)\right|_{x=a} \\
\left.D_{a^{+}}^{\alpha_{1}} \sin (k x)\right|_{x=b}
\end{array}\right.
$$

$\left.\begin{array}{c}\left.\mu_{1} D_{b^{-}}^{\beta_{1}} \cos (k x)\right|_{x=a}+\left.\mu_{2} D_{b^{-}}^{\beta_{2}} \cos (k x)\right|_{x=a} \\ \left.D_{a^{+}}^{\alpha_{1}} \cos (k x)\right|_{x=b}\end{array}\right]^{-1} \cdot\left[\begin{array}{l}L_{1} \\ L_{2}\end{array}\right]$

from which we can easily determine the constants of integration $C_{1}$ and $C_{2}$.

Remark 10. One can note that the fractional boundary conditions $\left.D_{b^{-}}^{\beta} y(x)\right|_{x=a}$ and $\left.D_{a^{+}}^{\alpha} y(x)\right|_{x=b}$ for integral values of parameters $\alpha$ and $\beta$ take the classical forms of boundary conditions; this means $\left.D_{b^{-}}^{n} y(x)\right|_{x=a} \equiv(-1)^{n} y^{(n)}(a)$ and $\left.D_{a^{+}}^{n} y(x)\right|_{x=b} \equiv y^{(n)}(b)$. In particular, it should be noted that the difference occurs, among others, in the boundary condition $\left.D_{b^{-}}^{1} y(x)\right|_{x=a} \equiv-y^{\prime}(a)$ and this form should be taken into account.

\section{Example of Solutions}

On the basis of the proposed method, to find the particular solutions to the considered equations (4) and (23), we calculated constants of integration $C_{1}$ and $C_{2}$ occurring in the general solutions that satisfy the sets of the given initial or boundary conditions (various combinations). In Figures 1-3, numerous examples of solutions have been presented.

\section{Conclusions}

The initial/boundary value problem for the second-order homogeneous differential equations with constant coefficients has been considered. The general solutions to these
Example 9. Equation (4) with the following set of boundary conditions:

$$
\begin{aligned}
& \left.\mu_{1} D_{b^{-}}^{\beta_{1}} y(x)\right|_{x=a}+\left.\mu_{2} D_{b^{-}}^{\beta_{2}} y(x)\right|_{x=a} \\
& =\left.L_{1} \wedge D_{a^{+}}^{\alpha_{1}} y(x)\right|_{x=b}=L_{2}, \\
& \qquad \mu_{1}, \mu_{2} \in \mathbb{R}, \quad\left|\mu_{1}\right|+\left|\mu_{2}\right|>0 .
\end{aligned}
$$

This case corresponds to the generalisation of the Robin boundary condition given at $x=a$. Also here we put general solution (5) into (41) and we obtain

$$
\begin{aligned}
& \mu_{1}\left(\left.C_{1} D_{b^{-}}^{\beta_{1}} \sin (k x)\right|_{x=a}+\left.C_{2} D_{b^{-}}^{\beta_{1}} \cos (k x)\right|_{x=a}\right) \\
& \quad+\mu_{2}\left(\left.C_{1} D_{b^{-}}^{\beta_{2}} \sin (k x)\right|_{x=a}+\left.C_{2} D_{b^{-}}^{\beta_{2}} \cos (k x)\right|_{x=a}\right) \\
& \quad=L_{1} \\
& \left.C_{1} D_{a^{+}}^{\alpha_{1}} \sin (k x)\right|_{x=b}+\left.C_{2} D_{a^{+}}^{\alpha_{1}} \cos (k x)\right|_{x=b}=L_{2}
\end{aligned}
$$

or

equations are widely known and involve arbitrary constants. Our aim was to find the particular solutions to this problem which satisfy the generalised boundary conditions. Such boundary conditions complement the set of classic boundary conditions (including the Dirichlet, Neumann, and Robin types) by including the fractional ones.

The use of the fractional boundary conditions in the considered initial/boundary value problem required the fractional differentiation of the general solutions. We derived the formulas for the left- and right-sided Riemann-Liouville fractional derivatives of the sine, cosine, hyperbolic sine, and hyperbolic cosine functions that occur in the general solutions. On this basis, the integration constants in these solutions were determined analytically.

On the plots, one can observe that the obtained results for the fractional boundary conditions are located between the solutions to the considered problem with respect to the classical (integer order) boundary conditions. Such behaviour of the particular solutions gives new possibilities in physical phenomena modelling, like the harmonic oscillator modelling, among others. In the future, we plan to apply this approach to seek solutions to other types of the initial/boundary value problems, in particular to the four-order problems.

\section{Conflicts of Interest}

The authors declare that they have no conflicts of interest. 

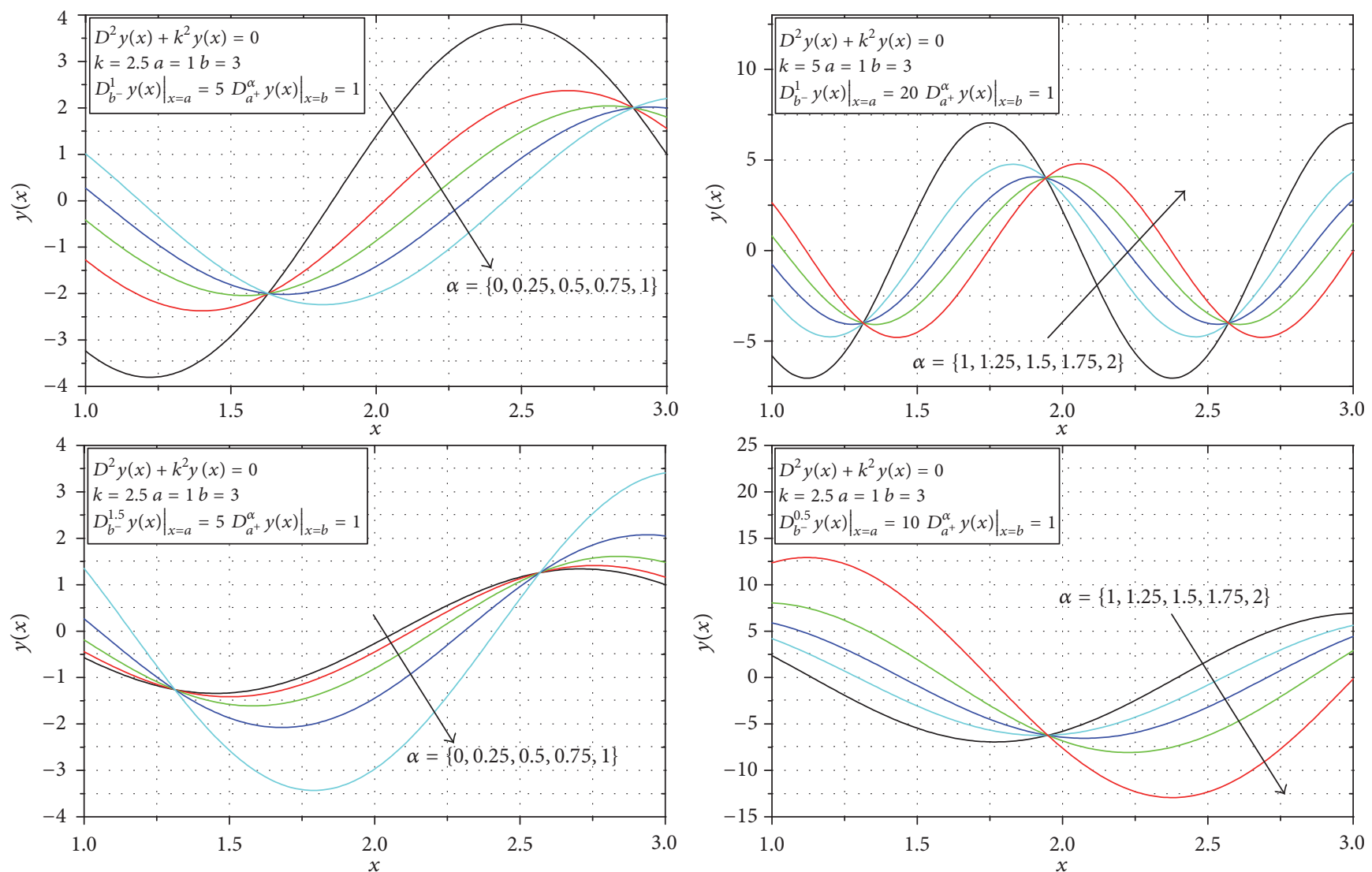

FIGURE 1: The particular solutions of (4) for selected boundary conditions (see details in the legends).
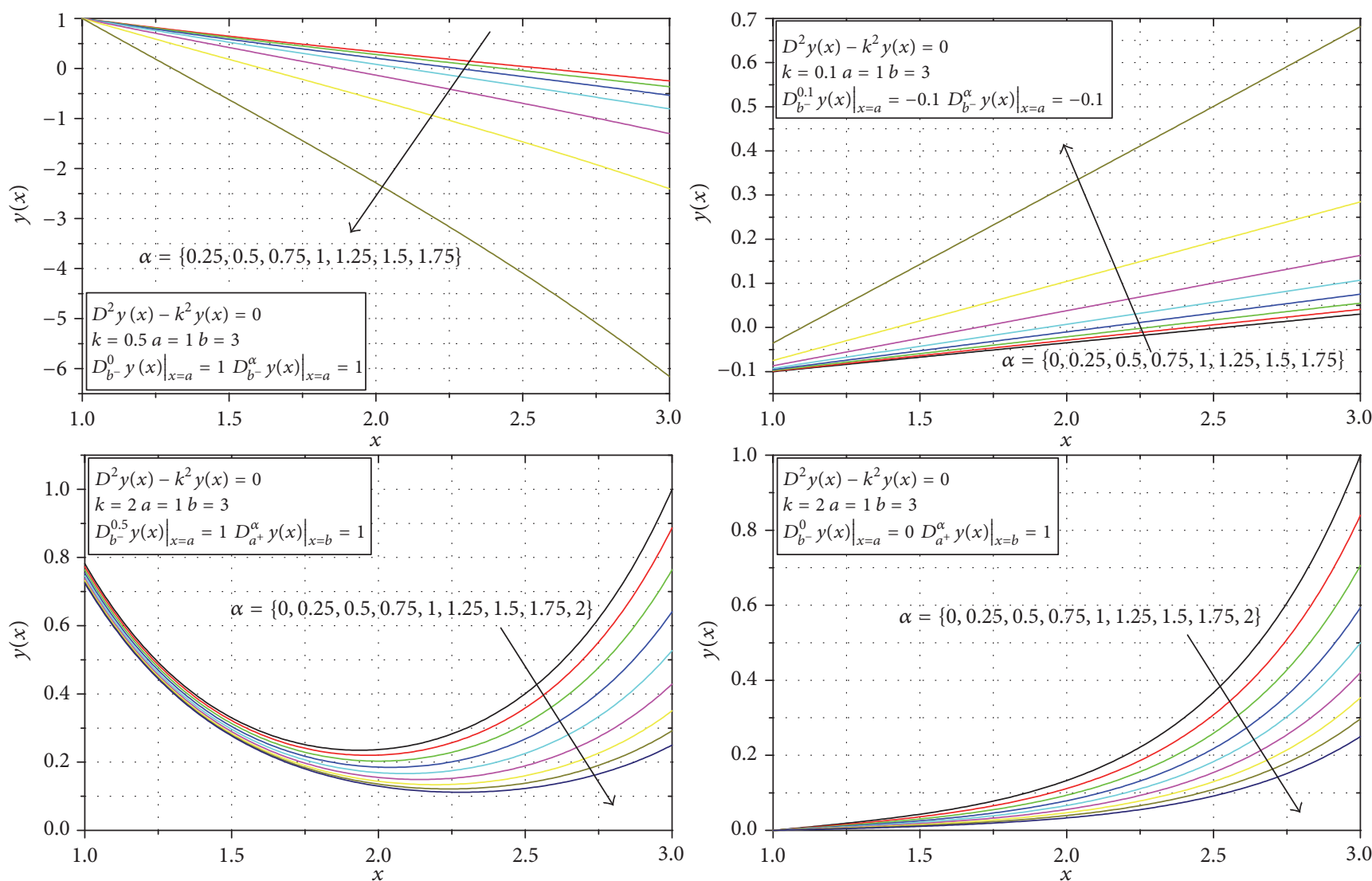

FIgURE 2: The particular solutions of (23) for selected boundary conditions (see details in the legends). 

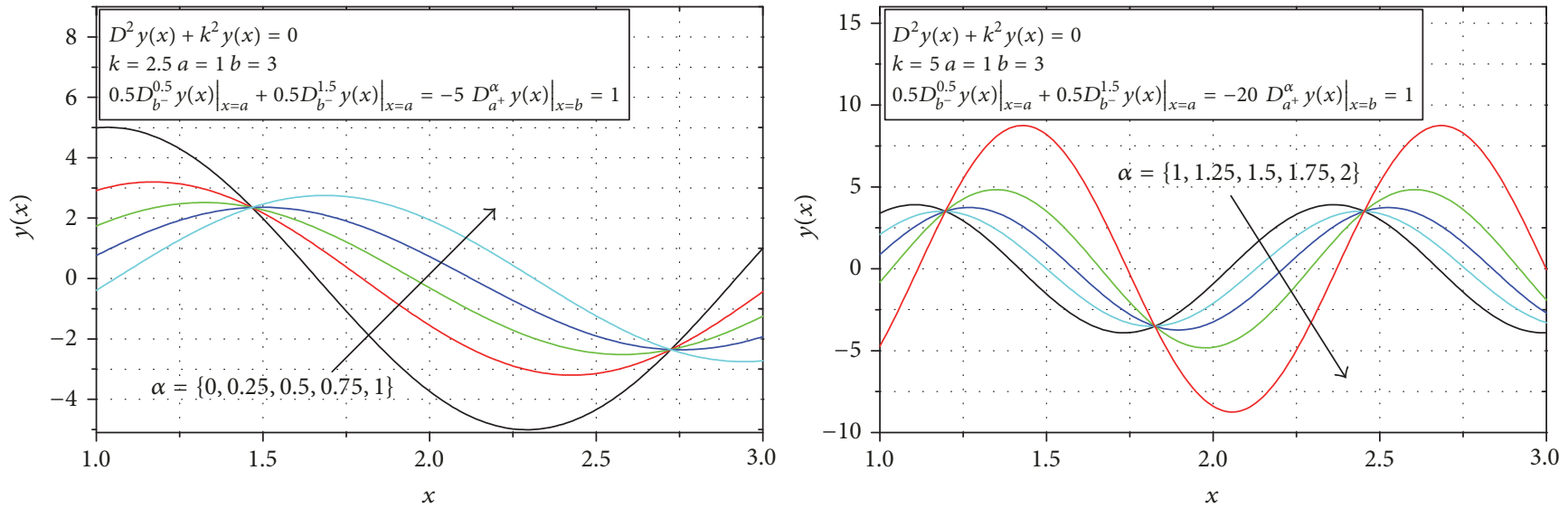

FIGURE 3: The particular solutions of (4) for selected boundary conditions (see details in the legends).

\section{Acknowledgments}

The research is supported by Faculty of Mechanical Engineering and Computer Science, Czestochowa University of Technology.

\section{References}

[1] M. J. Brennan and I. Kovaci, "Examples of physical systems described by the Duffing equation," in The Duffing Equation: Nonlinear Oscillators and their Behaviour, I. Kovacic and M. J. Brennan, Eds., John Wiley \& Sons, Ltd., 2011.

[2] A. Parmeggiani, Spectral Theory of Non-Commutative Harmonic Oscillators: An Introduction, vol. 1992 of Lecture Notes in Mathematics, Springer-Verlag, Berlin, Germany, 2010.

[3] P. Blaise and O. Henri-Rousseau, Quantum Oscillators, John Wiley \& Sons, Inc., Hoboken, NJ, USA, 2011.

[4] T. Pavlidis, Biological Oscillators: Their Mathematical Analysis, Academic Press, New York, NY, USA and London, UK, 1973.

[5] U. L. Rohde, A. K. Poddar, and G. Bock, The Design of Modern Microwave Oscillators for Wireless Applications: Theory and Optimization, John Wiley \& Sons, Inc., Hoboken, NJ, USA, 2005.

[6] C. Floris, "Stochastic stability of damped Mathieu oscillator parametrically excited by a gaussian noise," Mathematical Problems in Engineering, vol. 2012, Article ID 375913, 18 pages, 2012.

[7] L. S. Osborne, "A relativistic oscillator model applied to leptonnucleon reactions," Physics Letters B, vol. 63, no. 4, pp. 456-458, 1976.

[8] J. M. Cornwall and G. Tiktopoulos, "Functional Schrödinger equation approach to high-energy multiparticle scattering," Physics Letters B, vol. 282, no. 1-2, pp. 195-200, 1992.

[9] B. N. Achar, J. W. Hanneken, T. Enck, and T. Clarke, "Dynamics of the fractional oscillator," Physica A: Statistical Mechanics and its Applications, vol. 297, no. 3-4, pp. 361-367, 2001.

[10] B. N. Narahari Achar, J. W. Hanneken, and T. Clarke, "Damping characteristics of a fractional oscillator," Physica A: Statistical Mechanics and its Applications, vol. 339, no. 3-4, pp. 311-319, 2004.

[11] Y. Kang and X. Zhang, "Some comparison of two fractional oscillators," Physica B: Condensed Matter, vol. 405, no. 1, pp. 369-373, 2010.
[12] S. Kukla and U. Siedlecka, "Fractional heat conduction in multilayer spherical bodies," Journal of Applied Mathematics and Computational Mechanics, vol. 15, no. 4, pp. 83-92, 2016.

[13] Y. Povstenko and J. Klekot, "The fundamental solutions to the central symmetric time-fractional heat conduction equation with heat absorption," Journal of Applied Mathematics and Computational Mechanics, vol. 16, no. 2, pp. 101-112, 2017.

[14] A. A. Stanislavsky, “Twist of fractional oscillations," Physica A: Statistical Mechanics and its Applications, vol. 354, no. 1-4, pp. 101-110, 2005.

[15] F. Riewe, "Nonconservative Lagrangian and Hamiltonian mechanics," Physical Review E: Statistical, Nonlinear, and Soft Matter Physics, vol. 53, no. 2, pp. 1890-1899, 1996.

[16] F. Riewe, "Mechanics with fractional derivatives," Physical Review E: Statistical, Nonlinear, and Soft Matter Physics, vol. 55, no. 3, part B, pp. 3581-3592, 1997.

[17] M. Klimek, "Fractional sequential mechanics-models with symmetric fractional derivative," Czechoslovak Journal of Physics, vol. 51, no. 12, pp. 1348-1354, 2001.

[18] M. Klimek, "Lagrangean and Hamiltonian fractional sequential mechanics," Czechoslovak Journal of Physics, vol. 52, no. 11, pp. 1247-1253, 2002.

[19] O. P. Agrawal, "Formulation of Euler-Lagrange equations for fractional variational problems," Journal of Mathematical Analysis and Applications, vol. 272, no. 1, pp. 368-379, 2002.

[20] O. P. Agrawal, "Fractional variational calculus and the transversality conditions," Journal of Physics A: Mathematical and General, vol. 39, no. 33, pp. 10375-10384, 2006.

[21] D. Baleanu, J. H. Asad, and I. Petras, "Fractional Bateman - Feshbach Tikochinsky oscillator," Communications in Theoretical Physics, vol. 61, no. 2, pp. 221-225, 2014.

[22] T. Blaszczyk, "A numerical solution of a fractional oscillator equation in a non-resisting medium with natural boundary conditions," Romanian Reports in Physics, vol. 67, no. 2, pp. 350$358,2015$.

[23] T. Blaszczyk and M. Ciesielski, "Fractional oscillator equation: analytical solution and algorithm for its approximate computation," Journal of Vibration and Control, vol. 22, no. 8, pp. 20452052, 2016.

[24] M. Ciesielski and T. Blaszczyk, "Numerical solution of nonhomogenous fractional oscillator equation in integral form," Journal of Theoretical and Applied Mechanics, vol. 53, no. 4, pp. 959-968, 2015. 
[25] W. Liu, M. Wang, and T. Shen, "Analysis of a class of nonlinear fractional differential models generated by impulsive effects," Boundary Value Problems, vol. 175, 2017.

[26] Y. Xu and O. P. Agrawal, "Models and numerical solutions of generalized oscillator equations," Journal of Vibration and Acoustics, vol. 136, no. 5, Article ID 051005, 2014.

[27] A. A. Kilbas, H. M. Srivastava, and J. J. Trujillo, Theory and Applications of Fractional Differential Equations, Elsevier, Amsterdam, The Netherlands, 2006.

[28] G. B. Arfken and H. J. Weber, Mathematical Methods for Physicists, Elsevier, London, UK, 6th edition, 2005. 


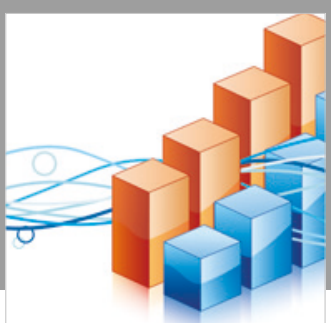

Advances in

Operations Research

\section{-n-m}
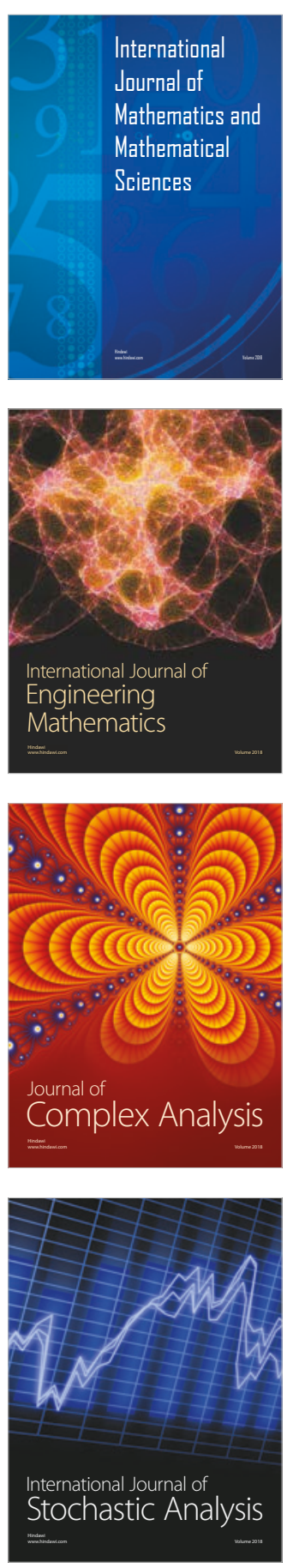
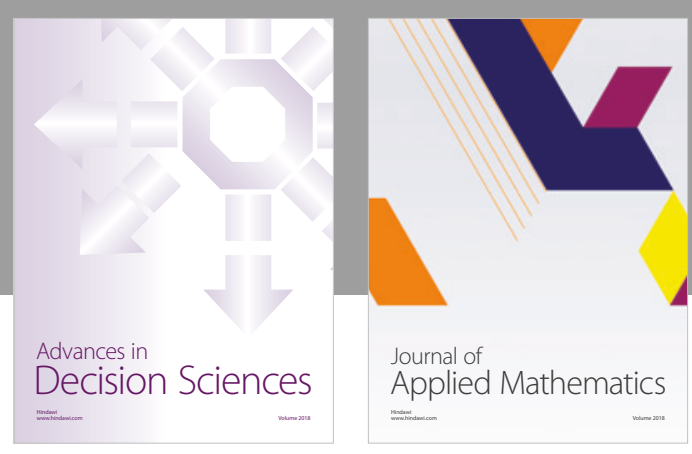

Journal of

Applied Mathematics
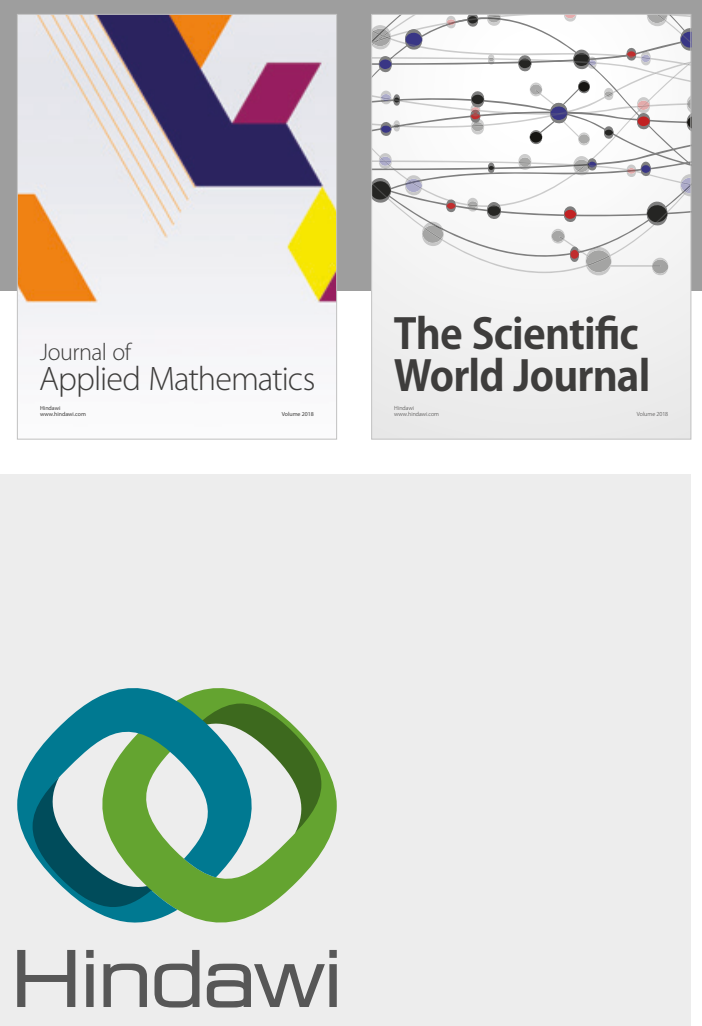

Submit your manuscripts at

www.hindawi.com

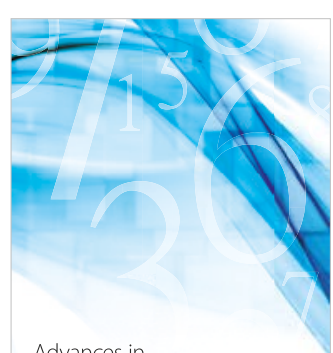

Advances in
Numerical Analysis
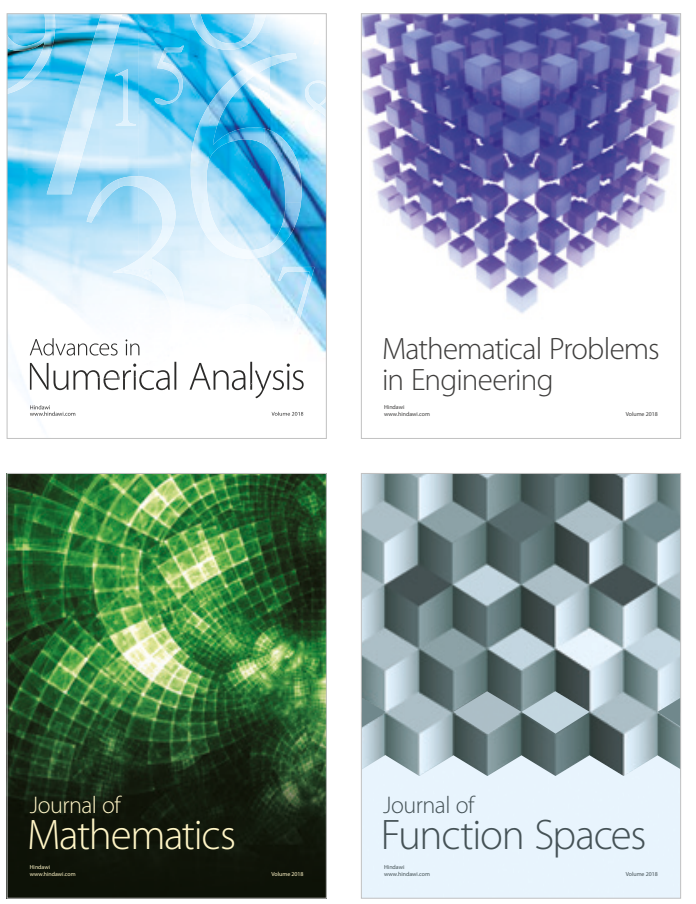

Mathematical Problems in Engineering

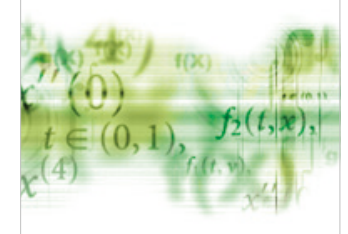

International Journal of

Differential Equations

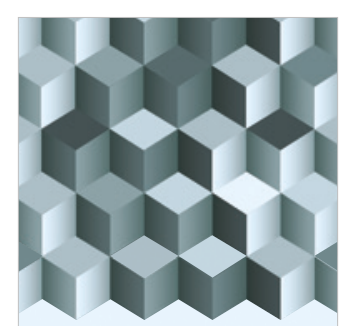

Journal of

Function Spaces

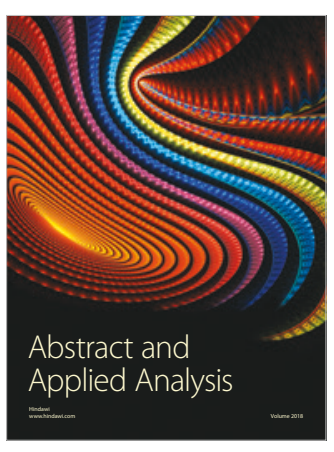

The Scientific

World Journal

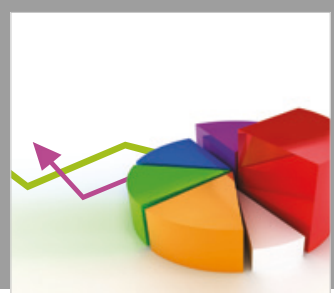

Journal of

Probability and Statistics
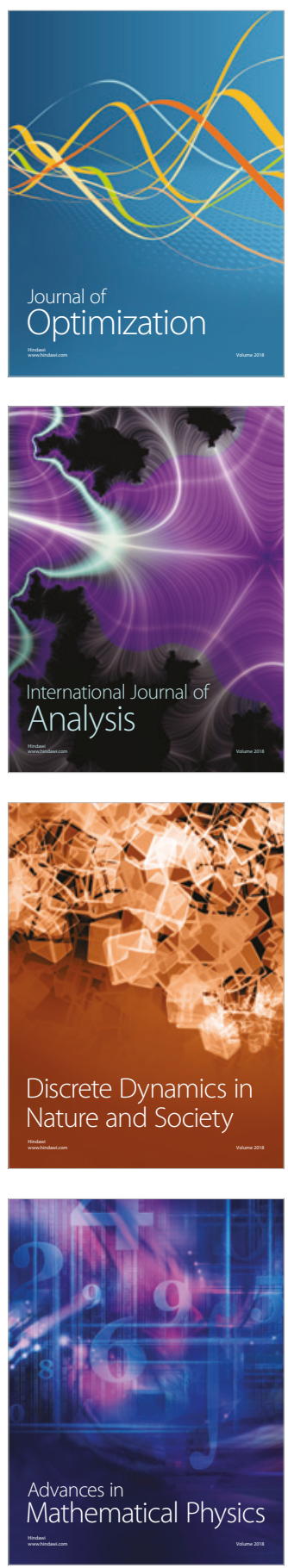